\title{
aiRock $^{\mathrm{TM}}$ : Reactive Transport Modeling Using High-Performance Computing in the Cloud and Machine Learning
}

\begin{abstract}
BABAK SHAFEI ${ }^{123}$
${ }^{1}$ AquaNRG Consulting Inc., 405 Main Street, Suite 807, Houston, TX 77002

We will present aiRock ${ }^{\mathrm{TM}}$, a state-of-the-art web application which applies high-performance computing (HPC) in the cloud and machine learning to estimate reactive flow properties in natural porous media such as aquifer's rock, sediments or soil. It leverages massive datasets acquired from high-resolution images obtained from scanning electron microscopy or X-ray computed tomography as well as results of an advanced multiscale reactive flow simulator.

Traditionally core-scale column experiments have been widely used in quest for measuring the fluid flow properties in porous media. The acquired data include porosity, permeability and water saturation that describe the physical properties of the rocks and fluids. Depending on the number of parameters of interest, column experiments can take weeks or even months to complete. Furthermore, the experiments are not carried out at in-situ subsurface conditions.

Recent advancements in high-resolution imaging techniques and HPC methods for physics-based simulations have introduced more accurate and cost-effective alternative solutions with high reproducibility of physical properties prediction of the rock samples compared to physical testing. Digital rock is an emerging technology which enhances the combination of high-resolution imaging and physics-based simulations to construct the equivalent virtual model of the column experiments. Applying digital rock physics techniques in multiphase flow systems (e.g. brine-gas) and large samples can be still computationally expensive. Moreover, the effects of geochemical parameters are ignored.

aiRock $^{\mathrm{TM}}$ offers a powerful, scalable and computationally inexpensive tool compared to Digital rock physics. This information provides valuable insights about the relationship between macroscopic flow properties such as permeability and extracted features from rock images. aiRock $^{\mathrm{TM}}$ enables the users to identify key physical-chemical factors such as wettability, mineralogy, porosity, connectivity and permeability that control fluid flow behavior.
\end{abstract}

